\title{
The POP-Q classification system: looking back and looking forward
}

\author{
Paul Riss • Peter L. Dwyer
}

Published online: 7 February 2014

(C) The International Urogynecological Association 2014

Pelvic organ prolapse is one of the most common problems facing every urogynecologist. Understandably, terminology is very important when describing vaginal or uterine descent, for two reasons. First, the physician's notes must represent the clinical problem so that the physician knows what has been seen and can relate it to a colleague. The second reason is quality assurance and research, where exact terminology is needed to accurately describe changes in pelvic organ prolapse and to be able to make comparisons between institutions and studies. In 1996 Bump et al. published a classification developed by several societies [1]. This POP-Q has been widely adopted and become the de facto standard in clinical medicine and research [2].

After almost 20 years we may ask whether this classification is set in stone or whether it needs modifying? Three areas should be considered:

1. The points and numbers of the POP-Q system are not intuitive and the choice of letters is often experienced as clumsy. Although simplifications of the POP-Q system have been proposed and seem to work [3] the fact remains that with a universal classification it is best to use the complete system. We ourselves have seen that it can be taught and learned without too much difficulty [4] and that with some practice clinicians soon become comfortable with the classification.

2. The staging system (1-4) seems like a carry-over from the time before the POP-Q system and may add confusion to

P. Riss $(\square)$

Gynecology and Obstetrics, Medical University of Vienna,

Anna Frauer Gasse 2, 1180 Vienna, Austria

e-mail: priuj11@gmail.com

P. L. Dwyer

Obstetrics and Gynecology, Mercy Hospital for Women,

University of Melbourne, Melbourne, Australia its proper use. Although clinicians like to use a staging system, pelvic organ prolapse cannot be described accurately by stages, and for this very reason the POP-Q was developed.

3. A description is what it is: letters and numbers to describe the result of an examination - nothing less and nothing more. Clinicians, however, have a tendency to immediately think of symptom severity and of treatment options. This is something that neither the POP-Q system nor the stages provide for.

The POP-Q system has without doubt become established as the premium classification and measurement of uterovaginal prolapse and is required by researchers who wish to publish in medical journals. However the use of multiple measurements taken at a number of vaginal sites is too detailed and complicated for many clinicians for everyday use. The POP-Q system does describe the position of various sites in the vagina; it does not specify what the prolapse is, namely whether it is bladder, cervix, enterocele sac or rectocele. Differentiating the type of prolapse is important because it directly relates to patient symptoms and their severity, what treatment is necessary and its effectiveness. For instance, repairing an enterocele alone will not improve bowel evacuation problems. The Baden-Walker half-way system does have a more simple $0-4$ grading system and classifies prolapse according to the defect site (urethra, bladder, cervix or cuff, pouch of Douglas, rectum and perineum, and descent above or below the vaginal introitus [5]. For this reason and because of its simplicity, the Baden-Walker system has maintained considerable popularity amongst gynecologists in their daily clinical practice.

Examination of prolapse, including taking measurements is usually performed in the left lateral, supine or sitting positions and rarely in the standing position, which will replicate most accurately the patient's prolapse. Also, the severity of POP 
varies in most patients over time; for instance, POP is worse toward the end of the day after standing or heavy lifting, and is best early morning after a night's rest. Thus, no POP classification will detect these changes in all situations. This seems unavoidable, but patients should be carefully questioned on the severity of the prolapse; and how far their prolapse protrudes and under what circumstances. For instance, does the prolapse descend to the introitus or beyond and how far beyond in centimeters (size of golf ball, tennis ball, etc.).

In this issue of the International Urogynecology Journal we present four articles highlighting these problem areas. Richard Bump gives us background information on how the POP-Q was developed 20 years ago [6]. The questions the committees had to deal with are still valid today, as we can see from the articles by Harmanli and Toozs-Hobson and Swift $[7,8]$. Dietz and Mann provide us with a correlation between the POP-Q system and prolapse symptoms from their own large database [9]. Should the stages be discarded altogether? Should they be reduced to three in number? What other modifications are needed with the points and numbers system? Should there be more or fewer points, e.g., addressing the lateral pelvic floor, to make use of the POP-Q system easier? What about point $D$ in the absence of a uterus? Would clinicians be able to accurately identify the insertion of the cardinal ligaments?

We are convinced that the POP-Q system represented a breakthrough in the accurate and reproducible description of pelvic organ prolapse and that it should be used as widely as possible in patient care and research. We also feel that the time has come for the standardization committees of our societies to take a new look at the POP-Q system. Whether this means that it should be changed remains to be seen.

\section{References}

1. Bump R, Mattiasson A, BO K, Brubaker L, DeLancey JOL, Klarskov P, Shull BL, Smith ARB (1996) The standardization of terminology of female pelvic organ prolapse and pelvic floor dysfunction. Am J Obstet Gynecol 175:10-17

2. Auwad W, Freeman RM, Swift S (2004) Is the pelvic organ prolapse quantification system (POPQ) being used? A survey of members of the International Continence Society (ICS) and the American Urogynecologic Society (AUGS). Int Urogynecol J Pelvic Floor Dysfunct 15(5):324-327

3. Manonai J, Mouritsen L, Palma P, Contreras-Ortiz O, Korte JE, Swift S (2011) The inter-system association between the simplified pelvic organ prolapse quantification system (S-POP) and the standard pelvic organ prolapse quantification system (POPQ) in describing pelvic organ prolapse. Int Urogynecol J 22(3):347-352. doi:10.1007/ s00192-010-1286-y

4. Geiss IM, Riss PA, Hanzal E, Dungl A (2007) A simple teaching tool for training the pelvic organ prolapse quantification system. Int Urogynecol J Pelvic Floor Dysfunct 18(9):1003-1005

5. Baden WF, Walker TA (1972) Physical diagnosis in the evaluation of vaginal relaxation. Clin Obstet Gynecol 15(4):1055-1069

6. Bump R. The POP-Q system - two decades of progress and debate. Int Urogynecol J. doi:10.1007/s00192-013-2262-0

7. Harmanli O. POP-Q 2.0: its time has come! Int Urogynecol J. doi:10.1007/s00192-013-2252-2

8. Toozs-Hobson P, Swift S. POP-Q stage I prolapse: is it time to alter our terminology? Int Urogynecol J. doi:10.1007/s00192-013-2260-2

9. Dietz HP, Mann KP. What is 'clinically relevant prolapse'? An attempt at defining cutoffs for the clinical assessment of pelvic organ descent. Int Urogynecol J. doi:10.1007/s00192-013-2307-4 